\title{
Validation of an Integrated Biomechanical Modeling Approach to the Ergonomic Evaluation of Drywall Installation
}

\author{
Lu Yuan \\ Department of Computer Science and Industrial Technology, Southeastern Louisiana \\ University, SLU 10847 Hammond, LA 70402 USA \\ Lu. Yuanaselu.edu
}

\begin{abstract}
The present study validated an integrated biomechanical modeling approach that the researcher has previously developed to study the physical demands for drywall installers. In particular, a sensitivity analysis was conducted to examine the impact of some quantitative assumptions that have been made to facilitate the modeling approach. Through setting up null hypothesis for each assumption and changing one parameter at a time, the new model output values were compared to the original ones. Using student t-tests to evaluate the statistical differences of the mean values, the sensitivity analysis was achieved by determining if any assumption or parameter has significant impact on the model. The results indicated that the modeling approach seemed to be the most sensitive to both the distribution of work cycles for a typical 8hour workday and the distribution and values of Euler angles that are used to determine the "shoulder rhythm." Whereas other assumptions including the distribution of trunk postures did not appear to have significant impact on the model output values. It was concluded that the integrated approach might provide an applicable examination of exposure variability particularly reflected by the non-routine feature of the work.
\end{abstract}

Keywords: Biomechanical Modeling, Sensitivity Analysis.

\section{Introduction}

Construction workers who perform drywall installation are at a high risk of various musculoskeletal injuries and disorders, especially to the low back and shoulder areas (Chiou et al., 2000; Hsiao and Stanevich, 1996; Lemasters et al., 1998; Lipscomb et al., 1997, 2000). Previous biomechanical analyses of drywall installation examined the physical stress and postural stability during lifting of the drywall panels (Pan and Chiou, 1999; Pan et al., 2002/2003). The authors were aware of many practical limitations to conducting accurate, non-invasive and reasonably priced ergonomic assessments at the worksite due to the dynamic nature of construction activities. Therefore, more reliable and cost-effective ergonomic exposure assessment methods are warranted. 
With the development and application of PATH (Posture, Activity, Tools, and Handling), an observational work sampling-based approach to direct observation (Buchholz et al., 1996), it has become practical to quantify the percent of time that construction workers are exposed to awkward postures, various tasks and activities, and manual handling (Buchholz et al., 2003; Forde and Buchholz, 2004; Paquet et al., 1999, 2001, 2005; Rosenberg et al., 2006). PATH has also been used in other industrial sectors that involve non-repetitive job activities including retail, agriculture, and healthcare industries (Earle-Richardson et al., 2005; Pan et al., 1999; Park et al., 2009).

The joint angle and load ranges that are represented by the PATH data are categorical rather than continuous. However, the Monte-Carlo simulation method, which is used to generate random numbers from a defined distribution, can be utilized to extract discrete values from the categorical PATH data for biomechanical analysis of the low back and shoulder (Tak et al., 2007). The Monte-Carlo method has also been successfully used both to capture the trunk muscle activity during torso bending (Mirka and Marras, 1993) and to simulate variability in muscle moment arms and physiological cross-sectional areas for prediction of shoulder muscle force (Chang et al., 2000; Hughes, 1997).

The researcher has previously explored a hybrid model integrating work sampling, computer simulation, and biomechanical modeling to conduct the ergonomic analysis of drywall installation (Yuan, 2006; Yuan et al., 2007). Since it is often infeasible to conduct direct measurements of ergonomic exposure assessment particularly within the construction industry, such a method has provided a reasonable alternative to estimate the physical demands during drywall installation. The present study was designed to demonstrate the validity and utility of this modeling approach, so that the impact of drywall storage position and size on the physical demands for drywall installers could be evaluated later. In particular, a sensitivity analysis was conducted to examine the impact of some quantitative assumptions that have been made to facilitate the modeling approach.

\section{Methods}

The integrated modeling approach started with the PATH methodology which provided the basic characterization of drywall installation work by quantifying the percent of time that the drywall installers were conducting different activities with different body segment (trunk, arm, and leg) postures. The relative frequencies of key activities, recorded over two hours, were used to construct the eight-hour-workday activity series using Monte-Carlo simulation. The biomechanical model input variables, including anthropometric data, joint angles, external load force and position vectors, and internal muscle parameters, were then generated for the analyses of the low back and shoulder. Utilizing different optimization programs in MATLAB (The MathWorks, Natick, MA, USA), the three-dimensional static equilibrium equations were solved and the biomechanical model output variables of muscle contraction forces and joint reaction forces at the low back and shoulder were computed. 
Seven main activities which represent a typical drywall installation task were examined in this study, including: 1. cut/measure; 2. lift; 3. carry; 4. hold/place; 5. screw; 6 . in between; and 7 . other. It was determined from the field observations that there were 12 possible work cycles, with 4 occurring during installation of a whole sheet and 8 denoting installation processes for a partial piece. The probability of each work cycle was calculated by multiplying the probability of every single activity during that cycle (Yuan, 2006). As studied by Pan and Chiou (1999), the drywall lifting method in which the worker used one hand to support the horizontal drywall sheet at its bottom and the other hand to grasp the sheet at its top produced the highest L4/L5 disc compression forces and therefore appeared to be the most stressful. It was assumed that the drywall installers in this study exclusively used such a lifting method as a demonstration of the worst case scenario. Activity 6 (in between) denoted exclusively loading/adjusting the screw guns and it always followed activity 5 (screw). Activity 7 (other) included climb/descend, communicate, mark/draw, and other miscellaneous job activities.

The drywall sheets studied in this project were Sheetrock® Brand Gypsum Panel from CGC Inc., with bulk density of $881 \mathrm{~kg} / \mathrm{m}^{3}\left(55 \mathrm{lb} / \mathrm{ft}^{3}\right)$. Summary statistics for subject weight, height, trunk widths and depths are acquired from Marras et al. (2001), because subject anthropometry was not obtained when the original PATH data were collected. The present study assumes subject height and weight follow a normal distribution because height and weight are generally known to be normally distributed (Roebuck et al., 1975). This assumption has been validated by Jung et al. (2009) using the 1988 US Army data (Gordon et al., 1988).

The relationships between subject trunk muscle parameters and anthropometric characteristics, such as subject height and weight, body mass index, and trunk width and depth at the planes of muscle origins and insertions, were determined by Marras et al. (2001). The regression equations with higher $\mathrm{R}$ squares were chosen in this study to represent those relationships. The weight percentages of different body segments of the whole body and the distance coefficients between the body segment center of mass and the proximal joint were calculated based on information from Drillis and Contini (1966) and Dempster (1955), respectively.

To conduct the sensitivity analysis, a list of the important quantitative assumptions that have been made to facilitate modeling was first created. These assumptions include, but are not limited to, the following:

1. The overall probabilities of the possible work cycles for a typical drywall installation task follow the general discrete distribution.

2. For a right-handed person, the trunk tends to lean toward the left side and twist counterclockwise for $80 \%$ of time during manual work (Tak et al., 2007).

3. Trunk flexion angles follow a lognormal distribution, whereas trunk lateral bend and twist angles both have normal distributions.

4. Values of the external load position on the Z-axis (directed upwards when the subject is standing erect) are dependent on the trunk flexion angles and show a normal distribution. 
5. Shoulder flexion angles display a normal distribution, and other motions of the shoulder, elbow, and wrist all have triangular distributions.

6. Values of Euler angles that are used to determine the "shoulder rhythm" (Högfors et al., 1991) have a normal distribution.

From this list, the researcher selected a small number of those assumptions (e.g., the probability of distribution type for trunk flexion angles) which are likely to have the biggest impact on the results for further examination. Through setting up the following null hypotheses for each assumption and changing one parameter at a time, the new model output values were compared to the original ones.

- Hypothesis 1: The distribution of work cycles for a typical 8-hour workday has significant impact on the low back model.

- Hypothesis 2: The probability of the trunk tending to lean toward the left side and twist counterclockwise for a right-handed person during manual work has significant impact on the low back model.

- Hypothesis 3: The distribution of trunk flexion angles has significant impact on the low back model.

- Hypothesis 4: The ranges of PATH trunk posture categories have significant impact on the low back model.

- Hypothesis 5: The distribution and values of Euler angles that are used to determine the "shoulder rhythm" have significant impact on the shoulder model.

Using student t-tests $(p<0.05)$ to evaluate the statistical differences of the means of major model input and output values including joint angles, muscle forces and joint reaction forces for the low back and shoulder for an average subject working on a typical 8-hour workday, the sensitivity analysis was achieved by determining if the model is sensitive to any assumption or parameter.

It should be noted that there is no gold standard for validation of the average muscle forces and joint reaction forces for a typical eight-hour workday. However, the results from a previous research study (Yuan, 2006) suggested that 1) the Monte Carlo simulation did generate the same activity distribution as the PATH observations; and 2) the output for the low back model gives similar results to the 3DSSPP (The University of Michigan, 1999).

\section{Results}

\subsection{Hypothesis 1: The Distribution of Work Cycles for a Typical 8-Hour Workday Has Significant Impact on the Low Back Model}

In the original model, the overall probabilities of the possible (12) work cycles for a typical 8-hour workday of drywall installation were assumed to follow the general discrete distribution. The present study examined the following two new assumptions and compared them with the original one: 
1. The 12 work cycles had equal probability; in other words, they follow the uniform distribution. And

2. The 4 work cycles for whole-sheet installation and the 8 work cycles for partialsheet installation had equal probability. This means that there is $1 / 8$ probability for each cycle of whole-sheet installation and 1/16 probability for each cycle of partial-sheet installation, respectively.

The comparison between the first assumption and the original one indicated that:

1. The ratio of activity 1 (cut/measure) over activity 2 (lift) was higher, which means that there were more work cycles of partial-sheet installation and the 8-hour workday tends to be less strenuous. However, the total work productivity (measured by the square foot of installation) might be reduced.

2. The angles of trunk flexion, lateral bending, and twisting were the same.

3. The MMCI (Maximum Muscle Contraction Intensity) and the forces of two major paired trunk muscles (Erector Spinae and Latissimus Dorsi) were statistically significantly smaller.

4. The absolute values of the L4/L5 joint reaction forces (disc compression, lateral shear, and anterior-posterior shear) were statistically significantly smaller $(\mathrm{t}=$ 5.715, $\mathrm{p}<0.0001 ; \mathrm{t}=2.414, \mathrm{p}=0.008$; and $\mathrm{t}=5.705, \mathrm{p}<0.0001$, respectively).

The comparison between the second assumption and the original one indicated that:

1. The frequency of the seven activities for a typical 8-hour workday appeared to be similar to that of the original model. And as a result,

2. There were no statistically significant differences among the joint angles, MMCI, muscle forces, and joint reaction forces.

Based on these findings, it appears that Hypothesis 1 could not be rejected. In other words, the distribution of work cycles for a typical 8-hour workday determines the frequency of the seven activities that were examined in this study; and consequently, has significant impact on the low back model output values.

\subsection{Hypothesis 2: The Probability of the Trunk Tending to Lean toward the Left Side and Twist Counterclockwise for a Right-Handed Person during Manual Work Has Significant Impact on the Low Back Model}

In the original model, it was assumed that "For a right-handed person, the trunk tends to lean toward the left side and twist counterclockwise for $80 \%$ of time during manual work." The present study changed such a probability to $50 \%$, 75\%, and $85 \%$, respectively. The comparisons between these probabilities and the original one indicated that there were no statistically significant differences among the MMCI and the forces of two major paired trunk muscles, as well as the absolute values of the L4/L5 joint reaction forces. Based on these examinations, it seems that Hypothesis 2 could be rejected. 


\subsection{Hypothesis 3: The Distribution of Trunk Flexion Angles has Significant Impact on the Low Back Model}

Trunk flexion angles were assumed to follow lognormal distribution in the original model. The researcher changed it to normal distribution and the comparison between those two indicated that there were no statistically significant differences among the model input and output values. This could suggest that Hypothesis 3 be rejected.

\subsection{Hypothesis 4: The Ranges of Path Trunk Posture Categories Have Significant Impact on the Low Back Model}

The comparison between those two ranges did not yield statistically significant differences on the model input and output values, except for the absolute values of the L4/L5 lateral shear force $(t=4.264, \mathrm{p}<0.0001)$. The forces of two major paired trunk muscles tended to be lower, although the differences were not statistically significant.

It seems that Hypothesis 4 could be rejected in general; however, further analysis might be needed to find out why the L4/L5 lateral shear forces were statistically different.

\subsection{Hypothesis 5: The Distribution and Values of Euler Angles That are Used to Determine the "Shoulder Rhythm" Have Significant Impact on the Shoulder Model}

In the original shoulder model, the means and standard deviations of the Euler angles were obtained independently from the ranges of such angles for three subjects in the motion studies conducted by Högfors et al. (1991). Through the review of a modified shoulder rhythm model by Makhsous (1999), the researcher was able to find the following equations describing the estimates for the Euler angles for the clavicle and scapula in accordance to the humerus.

$$
\begin{aligned}
& \alpha_{c}=-35.15+11.15 \cos \left[0.75\left(\beta_{h}+90\right)\right]\left(0.08 \alpha_{h}\right) \\
& \beta_{c}=18\left\{1-\cos \left[0.8\left(\beta_{h}+90\right)\right]\right\}+9 \\
& \gamma_{c}=30\left\{1-\cos \left[0.75\left(\beta_{h}+90\right)\right]\right\}+3 \\
& \alpha_{s}=200+20 \cos \left[0.75\left(\beta_{h}+90\right)\right] \\
& \beta_{s}=-87+42 \cos \left[-0.75 \beta_{h}-70\right]\left(0.1 \gamma_{h} / 90+1\right) \\
& \gamma_{s}=82+8 \cos \left\{\left(\alpha_{h}+10\right) \sin \left[0.75\left(\beta_{h}+90\right)\right]\right\}
\end{aligned}
$$

The new Euler angles seem to be different from the old ones, especially the values of $\beta_{\text {Scapula }}$. The comparison of the model output values indicated that the majority of muscle forces tended to be significantly bigger; whereas the coracohumeral ligament force was significantly smaller $(\mathrm{t}=8.738, \mathrm{p}<0.0001)$. On the other hand, the glenohumeral and sternoclavicular joint reaction forces were larger but the increases were not statistically significant, compared to those yielded from the original model. 
Based on these comparisons, it seems that Hypothesis 6 should not be rejected. As there is still a strong need to continue studying the "shoulder rhythm," the examination of Hypothesis 6 might indicate that Euler angles are indeed crucial in term of the understanding of the shoulder model.

\section{Discussion}

The present study validated an integrated biomechanical modeling approach that was previously developed by the researcher to conduct the ergonomic evaluation of drywall installation through a sensitivity analysis. The results indicated that both the distribution of work cycles for a typical 8-hour workday and the distribution and values of Euler angles that are used to determine the "shoulder rhythm" seemed to have the most significant impact on the modeling approach. Whereas other assumptions including the distribution of trunk postures did not appear to have significant impact on the model output values.

This study extracted information from PATH data and applied it to the simulation of a hypothetical subject. It was noteworthy that PATH observations were made on a crew of eight workers over two hours. As it is desired to determine the most efficient number of worker participating in the study relative to the number of repeated measurements for each of these workers (Van der Beek and Frings-Dresen, 1998), there might be other work cycles in reality besides the 12 that were analyzed. Yet, for a simplified simulation of a typical 8-hour workday, only these 12 work cycles were examined. Similarly, the activity sequences for the observed drywall installation work were able to be established to allow the generation of eight-hour-workday activity series. The acceptance of Hypothesis 1 in the present study suggested that these activity sequences eventually determine both the overall probabilities of the 12 work cycles and the low back model output values.

Because most construction work involves non-routine activities, it is not uncommon to see that there are certain patterns/sequences that construction workers usually follow in order to finish the work. For example, ironworkers would have to put the rebar in place before tying it. The method in this study can be used for the evaluation of those types of construction work. However, for some other types of construction work, the activity sequence may not be necessarily fixed: e.g., laborers will have to do many miscellaneous activities based on project schedule and needs. Also, it may be difficult to identify all possible activity series. Thus, comprehensive understanding and capturing of work are always required and it may also involve more reasonable assumptions in order for a realistic simulation of cumulative activities.

In order to generate random numbers from the observational data categories, many assumptions had to be made in terms of distribution type and pertinent parameters. Particularly, different distributional assumptions about body part postures were made, due to a lack of information on how body postures change over time even when repeating the same job activities (Tak et al., 2007). As Tak et al. (2007) has tested the validity of the simulation model as a whole, the sensitivity analysis of the impact of 
the trunk postures on the low back model in this study might have provided some useful information about the validation of the integrated modeling approach. The rejection of Hypotheses 2, 3, and 4 indicated that the different probabilities, types, and ranges of parameter distributions did not affect the results significantly.

On the other hand, it seems that there is lack of sufficient information regarding the shoulder model input values, particularly on the distributions of joint (shoulder, elbow and wrist) angles and the Euler angles that determine the "shoulder rhythm." Because of these limitations, the results of the shoulder model sensitivity analysis were less significant than those of the low back analysis; and thus, may need further evaluation and verification.

As construction work always involves a variety of activities that incur different biomechanical demands, it is imperative to determine the probabilistic representation of biomechanical stress in order to understand both acute and cumulative trauma risk (Mirka et al., 2000). Mirka et al. (2000) developed the Continuous Assessment of Back Stress (CABS) method by estimating the time-weighted distribution of biomechanical stress throughout the workday, therefore providing an important insight into some of the activities that would have been neglected using traditional task analysis methods. The present study explored similar idea by examining the continuous physical loads on the drywall installers' low back and shoulder. In contrast to Mirka et al. (2000), it considered activity sequences for the purpose of studying muscle fatigue characteristics (Yuan, 2006).

Overall, the present study attempted to integrate observational work-sampling, computer simulation, and biomechanical modeling for ergonomic exposure assessment in a typical construction drywall installation work, where it is infeasible to perform direct measurement in the field. The results of the sensitivity analysis implied that such integration might provide an applicable examination of exposure variability particularly reflected by the non-routine feature of the work.

Acknowledgments. The researcher thanks Dr. Bryan Buchholz of University of Massachusetts Lowell for providing guidance on the biomechanical modeling and Dr. Laura Welch of CPWR (Center for Construction Research and Training) for providing information on drywall installation tasks and activities. This study was supported by CPWR through NIOSH cooperative agreement OH009762. Its contents are solely the responsibility of the authors and do not necessarily represent the official views of CPWR or NIOSH.

\section{References}

Bean, J.C., Chaffin, D.B., Schultz, A.: Biomechanical model calculation of muscle contraction forces: a double linear programming method. Journal of Biomechanics 21(1), 59-66 (1988)

Buchholz, B., Paquet, V.L., Punnett, L., Lee, D., Moir, S.: PATH: A work sampling-based approach to ergonomic job analysis for construction and other non-repetitive work. Applied Ergonomics 27(3), 177-187 (1996) 
Buchholz, B., Paquet, V.L., Wellman, H., Forde, M.: Quantification of ergonomic hazards for ironworkers performing concrete reinforcement tasks during heavy highway construction. American Industrial Hygiene Association Journal 64(2), 243-250 (2003)

Chang, Y.W., Hughes, R.E., Su, F.C., Itoi, E., An, K.: Prediction of muscle force involved in shoulder internal rotation. Journal of Shoulder and Elbow Surgery 9(3), 188-195 (2000)

Chiou, S.S., Pan, C.S., Keane, P.: Traumatic injury among drywall installers, 1992 to 1995. Journal of Occupational and Environmental Medicine 42(11), 1101-1108 (2000)

Dempster, W.T.: Space requirements of the seated operator. WADC-TR-55-159, Aerospace Medical Research Laboratories, Dayton, Ohio (1955)

Drillis, R., Contini, R.: Body segment parameters, BP174-945. Tech. Rep. No. 1166.03, School of Engineering and Science, New York University, New York (1966)

Earle-Richardson, G., Jenkins, P., Fulmer, S., Mason, C., Burdick, P., May, J.: An ergonomic intervention to reduce back strain among apple harvest workers in New York State. Applied Ergonomics 36(3), 327-334 (2005)

Forde, M., Buchholz, B.: Task content and physical ergonomic risk factors in construction ironwork. International Journal of Industrial Ergonomics 34(4), 319-333 (2004)

Gordon, C.C., Bradtmiller, B., Churchill, Y., Clauser, C.E., McConville, J.T., Tebbetts, I.O., Walker, R.A.: Anthropometric Survey of U.S. Army [data file]. Available from National Technical Information Service Website (1988), http: / / www .ntis.gov

Högfors, C., Peterson, B., Sigholm, G., Herberts, P.: Biomechanical model of the human shoulder joint - II. The shoulder rhythm. Journal of Biomechanics 24(8), 699-709 (1991)

Hsiao, H., Stanevich, R.: Injuries and ergonomic applications in construction. In: Bhattacharya, A., McGlothlin, J.D. (eds.) Occupational Ergonomics, Theory and Applications, pp. 545-568. Marcel Dekker, Inc., New York (1996)

Hughes, R.E., An, K.: Monte Carlo simulation of a planar shoulder model. Medical \& Biological Engineering \& Computing 35, 544-548 (1997)

Jung, K., Kwon, O., You, H.: Development of a digital human model generation method for ergonomic design in virtual environment. International Journal of Industrial Ergonomics 39(5), 744-748 (2009)

Lemasters, G.K., Atterbury, M.R., Booth-Jones, A.D., Bhattacharya, A., Ollila-Glenn, N., Forrester, C., et al.: Prevalence of work related musculoskeletal disorders in active union carpenters. Occupational and Environmental Medicine 55, 421-427 (1998)

Lipscomb, H.J., Dement, J.M., Loomis, D.P., Silverstein, B., Kalat, J.: Surveillance of workrelated musculoskeletal injuries among union carpenters. American Journal of Industrial Medicine 32, 629-640 (1997)

Lipscomb, H.J., Dement, J.M., Gaal, J.S., Cameron, W., McDougall, V.: Work-related injuries in drywall installation. Applied Occupational and Environmental Hygiene 15(10), 794-802 (2000)

Makhsous, M.: Improvements, Validation and Adaptation of a Shoulder Model. Doctoral Dissertation, Chalmers University of Technology, Goteborg, Sweden (1999)

Marras, W.S., Jorgensen, M.J., Granata, K.P., Wiand, B.: Female and male trunk geometry: size and prediction of the spine loading trunk muscles derived from MRI. Clinical Biomechanics 16, 38-46 (2001)

Mirka, G.A., Marras, W.: A stochastic model of trunk muscle coactivation during trunk bending. Spine 18(11), 1396-1409 (1993)

Mirka, G.A., Kelaher, D.P., Nay, D.T., Lawrence, B.: Continuous Assessment of Back Stress (CABS): A new method to quantify low-back stress in jobs with variable biomechanical demands. Human Factors 42(2), 209-225 (2000) 
Pan, C.S., Gardner, L.I., Landsittel, D.P., Hendricks, S.A., Chiou, S.S., Punnett, L.: Ergonomic exposure assessment: an application of the PATH systematic observation method to retail workers. International Journal of Occupational and Environmental Health 2, $79-87$ (1999)

Pan, C.S., Chiou, S.: Analysis of biomechanical stresses during drywall lifting. International Journal of Industrial Ergonomics 23, 505-511 (1999)

Pan, C.S., Chiou, S.S., Hendricks, S.: The effect of drywall lifting method on workers' balance in a laboratory-based simulation. Occupational Ergonomics 3, 235-249 (2002/2003)

Paquet, V.L., Punnett, L., Buchholz, B.: An evaluation of manual materials handling in highway construction work. International Journal of Industrial Ergonomics 24, 431-444 (1999)

Paquet, V.L., Punnett, L., Buchholz, B.: Validity of fixed-interval observations for postural assessment in construction work. Applied Ergonomic 32, 215-224 (2001)

Paquet, V.L., Punnett, L., Woskie, S., Buchholz, B.: Reliable exposure assessment strategies for physical ergonomic stressors in construction and other non-routinized work. Ergonomics 48(9), 1200-1219 (2005)

Park, J.K., Boyer, J., Tessler, J., Casey, J., Schemm, L., Gore, R., Punnett, L.: Inter-rater reliability of PATH observations for assessment of ergonomic risk factors in hospital work. Ergonomics 52(7), 820-829 (2009)

Roebuck, J.A., Kroemer, K.H.E., Thomson, W.G.: Engineering anthropometry methods. Wiley-Interscience, New York (1975)

Rosenberg, B., Yuan, L., Fulmer, S.: Ergonomics of abrasive blasting: a comparison of high pressure water and steel shot. Applied Ergonomics 37, 659-667 (2006)

Tak, S., Punnett, L., Paquet, V., Woskie, S., Buchholz, B.: Estimation of compressive forces on lumbar spine from categorical posture data. Ergonomics 50(12), 2082-2094 (2007)

The University of Michigan, Center for Ergonomics. Three Dimensional Strength Prediction Program, v4.2: User's Manual (1999)

Van der Beek, A.J., Frings-Dresen, M.: Assessment of mechanical exposure in ergonomic epidemiology. Occupational and Environmental Medicine 55, 291-299 (1998)

Yuan, L.: Biomechanical analysis of the physical loads on the low back and shoulder during drywall installation. Doctoral Dissertation, University of Massachusetts Lowell, Lowell (2006)

Yuan, L., Buchholz, B., Punnett, L., Kriebel, D.: Estimation of muscle contraction forces and joint reaction forces at the low back and shoulder during drywall installation. In: Proceedings of the 51st Annual Meeting of Human Factors and Ergonomics Society, Baltimore, MD (2007) 